\title{
Review of: "Protein profiling of WERI RB1 and etoposide resistant WERI ETOR reveals new insights into topoisomerase inhibitor resistance in retinoblastoma"
}

\author{
Krishnakumar Subramanian
}

1 Sankara Nethralaya

Potential competing interests: The author(s) declared that no potential competing interests exist.

It is a straightforward paper comparing proteomic profiles of sensitive and resistant cell line of RB. But the value of it would have been clearer if they had shown what could be targeted in case of resistance. They have simply stopped at doing some pathway analysis and giving some loose inference. They should have used some inhibitor to block the pathways that they say are activated in resistant cells and show that they are important. That would provide some evidence of what could be targeted. They should do some more work. Not much novelty in this if they do not do functional studies.

Can they validate this ARHGAP9 a chemoresistance biomarker in RB identified in reference-1 Look at increased expression of neuronal stem cell markers - SOX2, NANOG, OCT4 and ABC transporters - ABCB1 and ABCC3. In resistant cell lines by immunocytochemistry or cell block preparation or blotting-reference-2 Look at the expression of some ECM related genes a or proteins in their data and validate-reference -3

1. Different transcriptome profiles between human retinoblastoma Y79 cells and an etoposide-resistant subline reveal a chemoresistance mechanism. Song WP, Zheng S, Yao HJ, Zhou XF, Li R, Zhang CY, Zhao JY, Wang LW, Shao RG, Li L.BMC Ophthalmol. 2020 Mar 6;20(1):92. doi: 10.1186/s12886-020-01348-6.

2. Balaji S, Santhi R, Kim U, Muthukkaruppan V, Priya CG, Vanniarajan A.Cancer Stem Cells with Overexpression of Neuronal Markers Enhance Chemoresistance and Invasion in Retinoblastoma. Curr Cancer Drug Targets. 2020;20(9):710-719. PMID: 32364077

3. Reinhard J, Wagner N, Krämer MM, Jarocki M, Joachim SC, Dick HB, Faissner A, Kakkassery VExpression Changes and Impact of the Extracellular Matrix on Etoposide Resistant Human Retinoblastoma Cell Lines... Int J Mol Sci. 2020 Jun 17;21(12):4322. doi: 10.3390/ijms21124322.PMID: 32560557 